TI 2012-092/II

Tinbergen Institute Discussion Paper
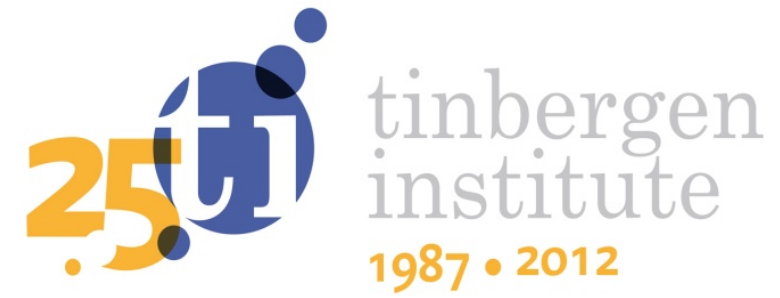

\title{
On Axiomatizations of the Shapley Value for Assignment Games
}

\author{
René van den Brink ${ }^{1}$ \\ Miklos Pinter ${ }^{2}$
}

I Faculty of Economics and Business Administration, VU University Amsterdam, and Tinbergen Institute;

2 Department of Mathematics, Corvinus University of Budapest. 


\title{
On axiomatizations of the Shapley value for assignment games*
}

\author{
René van den Brink† Miklós Pintér ${ }^{\ddagger}$
}

September 10, 2012

*Miklós Pintér gratefully acknowledges the Financial support by the Hungarian Scientific Research Fund (OTKA) and the János Bolyai Research Scholarship of the Hungarian Academy of Sciences.

${ }^{\dagger}$ VU University, Department of Econometrics and Tinbergen Institute, De Boelelaan 1105, 1081 HV Amsterdam,The Netherlands, jrbrink@feweb.vu.nl

$\ddagger$ Corvinus University of Budapest, Department of Mathematics and MTA-BCE "Lendület" Strategic Interactions Research Group, 1093 Hungary, Budapest, Fovám tér 13-15., miklos.pinter@uni-corvinus.hu 


\begin{abstract}
We consider the problem of axiomatizing the Shapley value on the class of assignment games. We first show that several axiomatizations of the Shapley value on the class of all TU-games do not characterize this solution on the class of assignment games by providing alternative solutions that satisfy these axioms. However, when considering an assignment game as a communication graph game where the game is simply the assignment game and the graph is a corresponding bipartite graph buyers are connected with sellers only, we show that Myerson's component efficiency and fairness axioms do characterize the Shapley value on the class of assignment games. Moreover, these two axioms have a natural interpretation for assignment games. Component efficiency yields submarket efficiency stating that the sum of the payoffs of all players in a submarket equals the worth of that submarket, where a submarket is a set of buyers and sellers such that all buyers in this set have zero valuation for the goods offered by the sellers outside the set, and all buyers outside the set have zero valuations for the goods offered by sellers inside the set. Fairness of the graph game solution boils down to valuation fairness stating that only changing the valuation of one particular buyer for the good offered by a particular seller changes the payoffs of this buyer and seller by the same amount.
\end{abstract}

Keywords: Assignment game, Shapley value, communication graph game, submarket efficiency, valuation fairness.

JEL code: C71, C78

\title{
1 Introduction
}

The history of assignment games goes back to the XIX. century to BöhmBawerk's [1] horse market model. Later Shapley and Shubik [21] introduced the formal, modern concept of assignment games.

One of the most popular solution concepts for TU-games is the Shapley value (Shapley [19]). Numerous axiomatizations of the Shapley value are known in the literature. In this paper we focus on the following ones: (1) Shapley's original axiomatization [19] by efficiency, the null player property (originally stated together as the carrier axiom), symmetry and additivity (also discussed by Dubey [6] and Peleg and Sudhölter [15]), (2) Young's [22] axiomatization replacing additivity and the null player property by strong monotonicity (also discussed by Moulin [12] and Pintér [16]), (3) Chun's [5] replacing strong monotonicity by coalitional strategic equivalence, (4) van 
den Brink's 2 2 replacing (in Shapley's original axiomatization) additivity and symmetry by fairness, and (5) Hart and Mas-Colell's [10] approaches using the potential function and a related reduced game consistency.

First, we examine these characterizations of the Shapley value on the class of assignment games, and conclude that none of these characterizations is valid on this class in the sense that they do not characterize a unique solution.

After this negative result we show that when considering an assignment game as a communication graph game where the game is simply the assignment game and the graph is a corresponding bipartite graph where buyers are connected with sellers only, Myerson [13]'s component efficiency and fairness axioms do characterize the Shapley value on the class of assignment games. Moreover, the axioms have a natural interpretation for these games.

An assignment game is fully described by the assignment situation being a set of buyers, a set of sellers, and for every buyer a valuation of the good offered by each seller. Instead of defining an assignment game as a communication graph game, we will directly work on the class of these assignment situations. For such assignment situations, component efficiency of a graph game solution boils down to submarket efficiency stating that the sum of the payoffs of all players in a submarket equals the worth of that submarket, where a submarket in an assignment situation is a set of buyers and sellers such that all buyers in this set have zero valuation for the goods offered by the sellers outside the set, and all buyers outside the set have zero valuations for the goods offered by sellers inside the set.

Fairness of the graph game solution boils down to valuation fairness stating that only changing the valuation of one particular buyer for the good offered by a particular seller changes the payoffs of this buyer and seller by the same amount. We show that these two axioms do characterize the Shapley solution for assignment situations being the solution that is obtained by applying the Shapley value to the corresponding assignment game. Since there is a one-to-one correspondence between assignment games and assignment situations on given sets of buyers and sellers, we will refer to the Shapley solution for assignment games simply as their Shapley value, and it follows that submarket efficiecy and valuation fairness also give an axiomatization of the Shapley value on the class of assignment games. So, we obtain a positive result by viewing an assignment game as a communication graph game.

Besides introducing and axiomatizing his solution, Myerson [13] also shows that it is stable for superadditive graph games in the sense that two players never get worse off when building a link between them. The Shapley value for assignment situations is stable in the sense that the payoffs of a buyer $i$ and seller $j$ do not decrease if only the valuation of buyer $i$ for the good offered by seller $j$ increases. 
The setup of the paper is as follows. Section 2 contains preliminaries. In Section 3 we apply the axiomatizations of the Shapley value for TU-games mentioned above to the class of assignment games, and show that they do not give uniqueness on this class. In Section 4 we consider assignment games as communication graph games and characterize the Shapley value for assignment situations by submarket efficiency and valuation fairness.

\section{Preliminaries}

\section{$2.1 \quad$ TU-games}

Let $N$ be a non-empty, finite set, let $|N|$ be its cardinality, and let $\mathcal{P}(N)$ denote the power set of $N$. A transferable utility (TU) game with player set $N$ is a pair $(N, v)$ with characteristic function $v: \mathcal{P}(N) \rightarrow \mathbb{R}$ such that $v(\emptyset)=0$. The class of all transferable utility games is denoted by $\mathcal{G}$, and the class of all characteristic functions on player set $N$ is denoted by $\mathcal{G}^{N}$. When there is no confusion about the player set we will often speak about game $v$ instead of game $(N, v)$. We define $\overline{\mathcal{G}}^{N}=\left\{(N, v) \mid v \in \mathcal{G}^{N}\right\}$.

It is well known that $\mathcal{G}^{N}$ is isomorphic with $\mathbb{R}^{2^{|N|}-1}$. Therefore we regard $\mathcal{G}^{N}$ and $\mathbb{R}^{2^{|N|}-1}$ as identical. Moreover, for $v \in \mathcal{G}^{N}$ and $\beta \in \mathbb{R}^{N}$, the game $v \oplus \beta \in \mathcal{G}^{N}$ is defined as $v \oplus \beta(S)=v(S)+\sum_{i \in S} \beta_{i}$ for all $S \subseteq N$, where $\beta_{i}$ is component $i$ of vector $\beta$.

For any $v \in \mathcal{G}^{N}, i \in N$ and $T \subseteq N$, let $m_{i}^{T}(v)=v(T \cup\{i\})-v(T)$ be player $i$ 's marginal contribution to coalition $T$ in game $v$. Obviously, $m_{i}^{T}(v)=0$ if $i \in T$.

Players $i, j \in N$ are symmetric in game $v \in \mathcal{G}^{N}$, if $m_{i}^{T}(v)=m_{j}^{T}(v)$ for all $T \subseteq N \backslash\{i, j\}$. Furthermore, we say that $i \in N$ is a null player in game $v$ if $m_{i}^{T}(v)=0$ for all $T \subseteq N$. The set of null players in game $v$ is denoted by $N P(v)$.

For $T \subseteq N, T \neq \emptyset$, the game $u_{T}$ given by $u_{T}(S)=1$ if $T \subseteq S$, and $u_{T}(S)=0$ otherwise, is called the unanimity game on coalition $T$. A game $\left(N, v_{0}\right)$ is a zero game if $v_{0}(T)=0$ for all $T \subseteq N$.

A (single-valued) solution on $\mathcal{C} \subseteq \mathcal{G}^{N}$, is a function $\phi: \mathcal{C} \rightarrow \mathbb{R}^{N}$. On $\mathcal{G}$ a solution is defined similar, assigning payoffs to any game on any player set $N$.

In this paper we focus on the Shapley value (Shapley [19]) $\phi^{S h}: \mathcal{G}^{N} \rightarrow \mathbb{R}^{N}$, for every $v \in \mathcal{G}^{N}$, given by

$$
\phi_{i}^{S h}(v)=\sum_{T \subseteq N \backslash\{i\}} \frac{|T| !(|N \backslash T|-1) !}{|N| !} m_{i}^{T}(v) \text { for all } i \in N .
$$


We refer to $\phi_{i}^{S h}(v)$ as the Shapley value of player $i$ in game $v \in \mathcal{G}^{N}$. On $\mathcal{G}$ the Shapley value is defined similar, assigning the above payoffs to any game on any player set $N$, but in case the player set is not obvious we write $\phi^{S h}(N, v)$ instead of $\phi^{\text {Sh }}(v)$.

For $v \in \mathcal{G}^{N}$ and $T \subseteq N, T \neq \emptyset$, the subgame of $v$ on $T, v^{T} \in \mathcal{G}^{T}$, is given by $v^{T}(S)=v(S)$ for all $S \subseteq T$.

Next, we recall some axioms that a solution can satisfy:

Solution $\phi$ on $\mathcal{C} \subseteq \mathcal{G}^{N}$

- is Pareto optimal (or efficient), if $\sum_{i \in N} \phi_{i}(v)=v(N)$ for all $v \in \mathcal{C}$;

- satisfies the null player property, if $\phi_{i}(v)=0$ for all $v \in \mathcal{C}$ and $i \in$ $N P(v)$;

- is anonymous, if $\phi(v) \circ \pi=\phi(v \circ \pi)$, for all $v \in \mathcal{C}$ and permutation $\pi$ on $N$ such that $v \circ \pi \in \mathcal{C}$;

- satisfies the equal treatment property, if $\phi_{i}(v)=\phi_{j}(v)$ for all $v \in \mathcal{C}$ and symmetric players $i, j$ in $v$;

- is covariant under strategic equivalence, if $\phi(\alpha v \oplus \beta)=\alpha \phi(v)+\beta$, for all $v \in \mathcal{C}, \alpha>0$ and $\beta \in \mathbb{R}^{N}$ such that $\alpha v \oplus \beta \in \mathcal{C}$;

- is additive, if $\phi(v+w)=\phi(v)+\phi(w)$ for all $v, w \in \mathcal{C}$ such that $v+w \in \mathcal{C}$;

- satisfies strong monotonicity, if $\phi_{i}(v) \leq \phi_{i}(w)$, for all $v, w \in \mathcal{C}$ and $i \in N$ such that $m_{i}^{T}(v) \leq m_{i}^{T}(w)$ for all $T \subseteq N$;

- satisfies marginality, if $\phi_{i}(v)=\phi_{i}(w)$, for all $v, w \in \mathcal{C}$ and $i \in N$ such that $m_{i}^{T}(v)=m_{i}^{T}(w)$ for all $T \subseteq N$;

- satisfies coalitional strategic equivalence $\mathrm{T}^{1}$ if $\phi_{i}(v)=\phi_{i}\left(v+\alpha u_{T}\right)$, for all $v \in \mathcal{C}, i \in N, T \subseteq N \backslash\{i\}$ and $\alpha>0$ such that $v+\alpha u_{T} \in \mathcal{C}$;

- satisfies fairness, if $\phi_{i}(v+w)-\phi_{i}(v)=\phi_{j}(v+w)-\phi_{j}(v)$ for all $v, w \in \mathcal{C}$ and $i, j \in N$ such that $i, j$ are symmetric in $w$ and $v+w \in \mathcal{C}$.

In order to discuss the approach of Hart and Mas-Colell [10] we need to consider classes of games that contain a player set $N$ and all its subsets. Let $\mathcal{C} \subseteq \mathcal{G}$ be such a class of games, $\phi$ be a solution on $\mathcal{C}$, and for all $(N, v) \in \mathcal{C}$, $T \subseteq N, T \neq \emptyset$, such that $\left(S \cup(N \backslash T), v^{S \cup(N \backslash T)}\right) \in \mathcal{C}$, let

\footnotetext{
${ }^{1}$ Notice that Chun's [4] original definition of this axiom is different from ours, but the two definitions are equivalent.
} 


$$
v_{T, \phi}(S)=v(S \cup(N \backslash T))-\sum_{i \in N \backslash T} \phi_{i}\left(v^{S \cup(N \backslash T)}\right) \text { for all } S \subseteq T, S \neq \emptyset,
$$

and $v_{T, \phi}(\emptyset)=0$. Then $v_{T, \phi} \in \mathcal{G}^{T}$ is called the $\phi$-reduced game of $v$ on coalition $T$. Solution $\phi$ defined on $\mathcal{C} \subseteq \mathcal{G}$

- is HM-consistent, briefly consistent, if for all $T \subseteq N, v \in \mathcal{C} \cap \mathcal{G}^{T}$ and $S \subseteq T, S \neq \emptyset$, such that $\left(S, v_{S, \phi}\right) \in \mathcal{C}$, it holds that $\phi_{i}\left(v_{S, \phi}\right)=\phi_{i}(v)$ for all $i \in S$.

From the literature it follows that the Shapley value is the unique solution on $\mathcal{G}^{N}$ that satisfies the following sets of axioms:

- Pareto optimality, the null player property, the equal treatment property and additivity (Shapley [19]);

- Pareto optimality, the equal treatment property and strong monotonicity (Young [22]);

- Pareto optimality, the equal treatment property and marginality (also by Young [22]);

- Pareto optimality, the equal treatment property and coalitional strategic equivalence (Chun [5]);

- Pareto optimality, the null player property and fairness (van den Brink [2]).

Further, it is the unique solution on $\mathcal{G}$ that satisfies $\mathrm{s}^{2}$

- Pareto optimality, covariance, the equal treatment property and consistency (Hart and Mas-Colell [10]).

\footnotetext{
${ }^{2}$ The definitions of the axioms given for classes $\mathcal{C} \subseteq \mathcal{G}^{N}$ are straightforwardly extended to classes $\mathcal{C} \subseteq \mathcal{G}$.
} 


\section{$2.2 \quad$ Assignment games}

Next we focus on the class of assignment games (Shapley and Shubik [21]). Let $B, S \subseteq N$ be two non-empty sets such that $B \cap S=\emptyset$ and $B \cup S=N$. The interpretation is the following, $B$ and $S$ are the sets of buyers and sellers, respectively. Every buyer wants one good, and every seller owns one good. These goods are not exactly the same, so a buyer can have different valuations for the goods owned by different sellers. We assume that the sellers have reservation value zero for every good. The nonnegative valuation (reservation value) of buyer $i \in B$ for the good offered by seller $j \in S$ is denoted by $a_{i, j} \geq 0$. So, buyer $i$ and seller $j$ can make a deal and earn worth $a_{i, j}$. Buyers cannot trade among each other (since they do not own a good), and also sellers cannot earn a worth among themselves since their valuation is zero.

Let $A$ be the $|B| \times|S|$ non-negative matrix with $a_{i, j}$ its $(i, j)$-th element. We refer to this matrix $A$ as an assignment situation or valuation matrix on $(B, S)$. We denote the collection of all assignment situations on $(B, S)$ by $\mathcal{A}^{B, S}$. Furthermore for all $T \subseteq N$, a matching on $T$ is a set of sets $M \subseteq\{\{i, j\} \subseteq T \mid i \in B \cap T, j \in S \cap T\}$ such that for every $g \in T$, $|\{\{h, k\} \in M \mid g \in\{h, k\}\}| \leq 1$. So, buyers can only be matched with sellers, sellers can only be matched with buyers, and every buyer (seller) can be matched with at most one seller (buyer). Let $\mathcal{M}(T)$ be the set of all matchings of $T$. Taking the sets of buyers $B$ and sellers $S$ fixed, the assignment game for valuation matrix $A$ is the game $v_{A}$ on $N=B \cup S$, given by ${ }^{3}$

$$
v_{A}(T)=\max _{M \in \mathcal{M}(T)} \sum_{\{i, j\} \in M} a_{i, j} \text { for all } T \subseteq B \cup S .
$$

Henceforth, let $\mathcal{G}^{B, S}$ be the class of assignment games with buyer and seller sets $B, S$. The elements of

$$
\underset{M \in \mathcal{M}(T)}{\arg \max } \sum_{\{i, j\} \in M} a_{i, j}
$$

are called the maximal matchings of coalition $T$. For any set of buyers and sellers $T$, the worth of this coalition is the maximum aggregated worth of the deals the involved players can achieve contingent on every player trading with at most one other player from the other type.

Since in the definition of assignment games, $B$ and $S$ are non-empty, in this paper every assignment game has at least two players. It is worth noting

\footnotetext{
${ }^{3}$ We use the convention that the empty sum is 0 .
} 
that if $u_{T} \in \mathcal{G}^{B, S}$ then $|T|=2$, and that for all $v \in \mathcal{G}^{B, S}$ and $\beta \in \mathbb{R}^{B \cup S}$ such that $v \oplus \beta \in \mathcal{G}^{B, S}, \beta=0$.

\subsection{Communication graph games}

Myerson [13] introduced a model in which it is assumed that the players in a game $v$ are part of a communication structure that is represented by an undirected graph $(N, L)$, with the player set $N$ as the set of nodes and $L \subseteq\{\{i, j\} \mid i, j \in N, i \neq j\}$ being a collection of edges or links, that is, subsets of $N$ such that each element of $L$ contains precisely two elements of $N$. Since in this paper the nodes in a graph represent the players in a game we use the same notation for the set of nodes as the set of players, and refer to the nodes in a graph just as players.

If there is no confusion about the player set $N$, we denote a graph on $N$ just by its set of links $L$ and refer to this as the graph. We denote the class of all possible sets of links on $N$ by $\mathcal{L}^{N}$. A sequence of $k$ different nodes $\left(i_{1}, \ldots, i_{k}\right)$ is a path between players $i_{1}$ and $i_{k}$ in $L \in \mathcal{L}^{N}$ if $\left\{i_{h}, i_{h+1}\right\} \in L$ for $h=1, \ldots, k-1$. A coalition $S \subseteq N$ is connected in graph $L$ if every pair of players in $S$ is connected by a path that only contains players from $S$, that is, for every $i, j \in S, i \neq j$, there is a path $\left(i_{1}, \ldots, i_{k}\right)$ such that $i_{1}=i, i_{k}=j$ and $\left\{i_{1}, \ldots, i_{k}\right\} \subseteq S$. Coalition $T \subseteq S$ is a component of $S$ in graph $L$ if it is a maximally connected subset of $S$, that is, $T$ is connected in $L(S)$ and for every $h \in S \backslash T$ the coalition $T \cup\{h\}$ is not connected in $L(S)$, where $L(S)=\{\{i, j\} \in L \mid\{i, j\} \subseteq S\}$. We denote the set of components of $S \subseteq N$ in $L$ by $C_{L}(S)$.

A pair $(v, L) \in \mathcal{G}^{N} \times \mathcal{L}^{N}$ is referred to as a graph game on $N$. Following Myerson [13], in the graph game $(v, L)$ players can cooperate if and only if they are able to communicate with each other, that is, a coalition $S$ can realize its worth $v(S)$ if and only if $S$ is connected in $L$. Whenever this is not the case, players in $S$ can only realize the sum of the worths of the components of $S$ in $L$. This yields the (Myerson) restricted game $v^{L} \in \mathcal{G}^{N}$ given by

$$
v^{L}(S)=\sum_{T \in C_{L}(S)} v(T), \quad S \subseteq N .
$$

The Myerson value is the solution $\mu: \mathcal{G}^{N} \times \mathcal{L}^{N} \rightarrow \mathbb{R}^{N}$ that is obtained by taking the Shapley value of the restricted game $v^{L}$, that is,

$$
\mu(v, L)=\phi^{S h}\left(v^{L}\right) \text {, for all } v \in \mathcal{G}^{N} \text { and } L \in \mathcal{L}^{N} .
$$


Besides introducing this value, Myerson [13] gives a characterization by the axioms of component efficiency and fairness. Solution $\phi$ on $\mathcal{C} \subseteq \mathcal{G}^{N} \times \mathcal{L}^{N}$ satisfies

- component efficiency, if for every graph game $(v, L) \in \mathcal{G}^{N} \times \mathcal{L}^{N}$ and component $S \in C_{L}(N)$, it holds that $\sum_{i \in S} \phi_{i}(v, L)=v(S)$;

- graph game fairness ${ }^{4}$ if for every graph game $(v, L) \in \mathcal{G}^{N} \times \mathcal{L}^{N}$ and pair of players $i, j \in N$, it holds that $\phi_{i}(v, L)-\phi_{i}(v, L \backslash\{i, j\})=$ $\phi_{j}(v, L)-\phi_{j}(v, L \backslash\{i, j\})$.

Component efficiency states that the sum of the payoffs of all players in a component equals the worth of that component. Graph game fairness states that deleting the link between two players changes their payoffs by the same amount. Moreover, Myerson [13] shows that his solution is stable in the sense that for superadditive games adding a link never hurts the two players incident with that link. Game $v$ is superadditive if $v(S \cup T) \geq v(S)+v(T)$ for all $S, T \subseteq N$ with $S \cap T=\emptyset$.

Theorem 2.1. (Myerson [13])

(i) The Myerson value is the unique solution on $\mathcal{G}^{N} \times \mathcal{L}^{N}$ that satisfies component efficiency and graph game fairness.

(ii) For each graph game $(v, L) \in \mathcal{G}^{N} \times \mathcal{L}^{N}$ with $v$ superadditive, it holds that $\mu_{i}(v, L) \geq \mu_{i}(v, L \backslash\{l\}), i \in l \in L$.

\section{Axioms of the Shapley value on the class of assignment games}

In this section we consider two basic types of axiomatizations of the Shapley value that are mentioned in Subsection 2.1 for the class of all TU-games, but now on the class of assignment games: the first where the player set is fixed, and the second where it is not fixed.

\subsection{Axiomatizations on a fixed player set}

We start this subsection with a slight and trivial, but positive result stating that for two-player assignment games, that is, a game with one seller and one buyer, there is only one solution satisfying Pareto optimality and the equal treatment property. (We omit the obvious proof.)

\footnotetext{
${ }^{4}$ Myerson [13] just refers to this as fairness, but we call it graph game fairness to distinguish it from TU-game fairness as discussed in Section 2.1
} 
Theorem 3.1. If $|B \cup S|=2$, then solution $\phi$ on $\mathcal{G}^{B, S}$ satisfies Pareto optimality and the equal treatment property if and only if it is the Shapley value.

Henceforth we assume that $|B \cup S|>2$, that is, the class of assignment games under consideration is not the "trivial" one. We show that none of the axiomatizations of the Shapley value on the class of all TU-games on a fixed player set mentioned before characterize the Shapley value on the class of assignment games. We do this by presenting another solution that satisfies the properties. Let $O R(N)$ be the set of all (linear) orderings on set $N$. Consider the following two solutions for assignment games with buyer and seller sets $B$ and $S$.

First, let

$$
O R_{B}=\{\tau \in O R(B \cup S)|\tau(i) \leq| B \mid \Rightarrow i \in B\},
$$

be the orders where the buyers come first, and let

$$
O R_{S}=\{\tau \in O R(B \cup S)|\tau(i) \leq| S \mid \Rightarrow i \in S\},
$$

be the orders where the sellers come first.

Now, for all $v \in \mathcal{G}^{B, S}$ and $i \in B \cup S$, let

$$
\begin{array}{r}
\phi_{i}^{B}(v)=\frac{1}{\left|O R_{B}\right|}\left(\sum_{\tau \in O R_{B}}(v(\{j \in B \cup S \mid \tau(j) \leq \tau(i)\})\right. \\
-v(\{j \in B \cup S \mid \tau(j)<\tau(i)\}))),
\end{array}
$$

be the average marginal contribution of buyer or seller $i$ over all orders where the buyers come first, and

$$
\begin{array}{r}
\phi_{i}^{S}(v)=\frac{1}{\left|O R_{S}\right|}\left(\sum_{\tau \in O R_{S}}(v(\{j \in B \cup S \mid \tau(j) \leq \tau(i)\})\right. \\
-v(\{j \in B \cup S \mid \tau(j)<\tau(i)\})))
\end{array}
$$

be the average marginal contribution of buyer or seller $i$ over all orders where the sellers come first. Then, for all $v \in \mathcal{G}^{B, S}$, let

$$
\phi^{B, S}(v)=\frac{\phi^{B}(v)+\phi^{S}(v)}{2}
$$


be the average of these two solutions. It turns out that $\phi^{B, S}$ also satisfies properties on the class of all assignment games that characterize the Shapley value on the class of all TU-games.

Proposition 3.2. Solution $\phi^{B, S}$ is a convex combination of random order values and satisfies anonymity, the equal treatment property, covariance, additivity, and strong monotonicity on $\mathcal{G}^{B, S}$.

Proof. It is easy to verify that $\phi^{B, S}$ is a convex combination of random order values that satisfies covariance, additivity and strong monotonicity. (The proof is left for the reader.)

Anonymity: Since the sets $B$ and $S$ are fixed, one can apply only permutations which permute buyer with buyer and seller with seller, otherwise sets $B$ and $S$ would change. Therefore, both solutions $\phi_{i}^{B}$ and $\phi_{i}^{S}$ satisfy anonymity, and so do their convex combinations.

Equal treatment property: If $i, j \in N$ are symmetric in game $v$, then there can be two cases:

(1) Suppose that both players are buyers or both are sellers. In this case anonymity implies the equal treatment property.

(2) Otherwise, suppose w.l.o.g. that $i \in B$ and $j \in S$. Then $a_{i, h}=0$ for all $h \in S \backslash\{j\}$ and $a_{h, j}=0$ for all $h \in B \backslash\{i\}$. Then $m_{i}^{T}(v)=0$ for all $T \subseteq N \backslash\{j\}$, and $m_{i}^{T}(v)=v(\{i, j\})$ for all $T \subseteq N, j \in T$. Similar, $m_{j}^{T}(v)=0$ for all $T \subseteq N \backslash\{i\}$, and $m_{j}^{T}(v)=v(\{i, j\})$ for all $T \subseteq N, i \in T$. Then $\phi_{i}^{B}(v)=0$ and $\phi_{j}^{B}(v)=v(\{i, j\})$. Similarly, $\phi_{i}^{S}(v)=v(\{i, j\})$ and $\phi_{j}^{S}(v)=0$. Therefore $\phi_{i}^{B, S}(v)=\phi_{j}^{B, S}(v)$.

Solution $\phi^{B, S}$ being different from the Shapley value on the class of assignment games follows from the following simple example.

Example 3.3. Consider $B=\{1,2\}, S=\{3\}$, and $v \in \mathcal{G}^{B, S}$ determined by $a_{1,3}=1$ and $a_{2,3}=2$, that is, $v(\{1,3\})=1, v(\{2,3\})=v(\{1,2,3\})=2$ and $v(T)=0$ otherwise. Then $\phi^{B}(v)=(0,0,2), \phi^{S}(v)=\left(\frac{1}{2}, \frac{3}{2}, 0\right)$, and thus $\phi^{B, S}(v)=\left(\frac{1}{4}, \frac{3}{4}, 1\right)$. However, $\phi^{S h}(v)=\left(\frac{1}{6}, \frac{2}{3}, \frac{7}{6}\right)$.

From Proposition 3.2 and Example 3.3, it follows that the in Subsection 2.1 mentioned axiomatizations of the Shapley value on $\mathcal{G}^{N}$ are not valid on $\mathcal{G}^{B, S}$ in the sense that the corresponding axioms do not give uniqueness..$^{5}$

Corollary 3.4. On the class $\mathcal{G}^{B, S}$ of assignment games,

\footnotetext{
${ }^{5}$ Here we also use that strong monotonicity implies marginality, marginality implies coalitional strategic equivalence, the equal treatment property and additivity imply fairness, and every random order value satisfies Pareto optimality and the null player property.
} 
- Shapley's axiomatization (Pareto optimality, the null player property, the equal treatment property (anonymity) and additivity),

- Young's axiomatization (Pareto optimality, the equal treatment property and strong monotonicity (marginality)),

- Chun's axiomatization (Pareto optimality, the equal treatment property and coalitional strategic equivalence) and

- van den Brink's axiomatization (Pareto optimality, the null player property and fairness),

of the Shapley value are not valid.

Remark 3.5. Roth [17] showed that the Shapley value can be interpreted as a von Neumann-Morgenstern utility function. His set-up can be adapted to the class of assignment games as well. However, since his result is closely connected to the axioms of Pareto optimality, the null player property, anonymity and additivity, that is, to Shapley's original axiomatization, this characterization is not valid on the class of non-trivial assignment games either.

Finally, we notice the following.

Remark 3.6. On the class of assignment games, coalitional strategic equivalence is strictly weaker than marginality, and marginality is strictly weaker than strong monotonicity.

\subsection{Variable player set}

In this subsection we consider Hart and Mas-Colell's [10] two approaches on a variable player set.

Definition 3.7. Let $\mathcal{C} \subseteq \mathcal{G}$. For every function $P: \mathcal{C} \rightarrow \mathbb{R}, T \subseteq N, T \neq \emptyset$, and for all $v \in \mathcal{G}^{T}$ such that $(T, v) \in \mathcal{C}$ and $i \in T$ such that $|T|=1$ or $\left(T \backslash\{i\}, v^{T \backslash\{i\}}\right) \in \mathcal{C}$, let

$$
P_{i}^{\prime}(v)= \begin{cases}P(v), & \text { if }|T|=1 \\ P(v)-P\left(v^{T \backslash\{i\}}\right) & \text { otherwise. }\end{cases}
$$

If

$$
\sum_{i \in T} P_{i}^{\prime}(v)=v(T)
$$

for all $v \in \mathcal{G}^{T}$ such that $(T, v) \in \mathcal{C}$ such that either $|T|=1$ or $(T \backslash$ $\left.\{i\}, v^{T \backslash\{i\}}\right) \in \mathcal{C}$ for all $i \in T$, then $P$ is called a potential on $\mathcal{C}$. 
Definition 3.8. A collection $\mathcal{C} \subseteq \mathcal{G}$ is subgame closed, if $\left(T \backslash\{i\}, v^{T \backslash\{i\}}\right) \in \mathcal{C}$ for all $T \subseteq N$ with $|T|>1, i \in T$ and $v \in \mathcal{G}^{T}$ such that $(T, v) \in \mathcal{C}$.

Since every game has at least one player, in the above definition we require that subgame $v^{T \backslash\{i\}}$ is in the set under consideration only if there are at least two players in $T$.

Proposition 3.9. Let $\mathcal{C} \subseteq \mathcal{G}$ be a subgame closed set of games. Then function $P$ on $\mathcal{C}$ is a potential, if and only if $P_{i}^{\prime}(v)=\phi_{i}^{S h}(v)$ for all $T \subseteq N$, $T \neq \emptyset, v \in \mathcal{G}^{T}$ such that $(T, v) \in \mathcal{C}$ and $i \in T$.

Proof. It comes directly from Hart and Mas-Colell [10] and Peleg and Sudhölter [15] Theorem 8.4.4. (pp. 216-217).

Next we look into the case of assignment games with at least one seller and buyer.

Corollary 3.10. On the class of assignment games there is a potential $P$ such that there exists an assignment game $v$ and a player $i$ such that $P_{i}^{\prime}(v) \neq$ $\phi_{i}^{S h}(v)$.

Proof. Let $B=\{i\}, S=\{j\}$ and $v \in \mathcal{G}^{B, S}$. In this case, neither $v^{(B \cup S) \backslash\{i\}}$ nor $v^{(B \cup S) \backslash\{j\}}$ are assignment games.

In general, the potential is not well defined on the class of assignment games with two players, therefore one can give any value to these games. Since the potential is defined recursively (see Definition 3.7), its value on any game is determined by these arbitrarily fixed values. Summing up, there are as many as continuum different potentials on the class of assignment games.

Remark 3.11. If we allow assignment games where the set of buyers and/or the set of sellers can be empty sets then Hart and Mas-Colell's potential function characterization becomes valid on this redefined class of assignment games.

Next we demonstrate that Hart and Mas-Colell's approach applying the axioms Pareto optimality, covariance, the equal treatment property and consistency, see Subsection 2.1, does not work on the class of assignment games either. We do this by defining the following solution.

First, let $\mathcal{G}_{a}$ be the collection of all assignment games, that is, $\mathcal{G}_{a}=\{(N, v) \in$ $\mathcal{G} \mid$ there exist $B, S \subset N, B \neq \emptyset, S \neq \emptyset, B \cap S=\emptyset, B \cup S=N$ such that $\left.(N, v) \in \mathcal{G}^{B, S}\right\}$. Moreover, let solution $\bar{\phi}$ on $\mathcal{G}_{a}$ for each $v \in \mathcal{G}_{a} \cap \mathcal{G}^{B, S}$ be given by 


$$
\bar{\phi}_{i}(v)= \begin{cases}\frac{v(B \cup S)}{|(B \cup S) \backslash N P(v)|}, & \text { if } i \notin N P(v) \\ 0 & \text { otherwise. }\end{cases}
$$

It is worth noticing that for any assignment game $v$ and player $i \notin N P(v)$, $\bar{\phi}_{i}(v)>0$.

Proposition 3.12. Solution $\bar{\phi}$ satisfies Pareto optimality, anonymity, the equal treatment property, covariance and consistency on $\mathcal{G}_{a}$.

Proof. It is left for the reader to verify that $\bar{\phi}$ satisfies Pareto optimality, anonymity and the equal treatment property.

Covariance follows since $v \oplus \beta \in \mathcal{G}_{a}$ implies that $\beta=0$.

Consistency: If $|B \cup S|=2$ then for all $v \in \mathcal{G}^{B, S}, \bar{\phi}(v)=\phi^{S h}(v)$, so we are done.

Let $|B \cup S|>2, v \in \mathcal{G}^{B, S}$ and $T \subseteq B \cup S$ be such that $v_{T, \bar{\phi}}$ is an assignment game. To prove that $\bar{\phi}_{i}\left(v_{T, \bar{\phi}}\right)=\bar{\phi}_{i}(v)$ for all $i \in T$, it is sufficient to prove that for all $i \in T$ it holds that: $i \in N P\left(v_{T, \bar{\phi}}\right)$ if and only if $i \in N P(v)$. First, suppose that $i \in N P(v)$. Then $v(S \cup\{i\} \cup((B \cup S) \backslash T))=v(S \cup((B \cup S) \backslash T))$ for all $S \subseteq T$. But then $v_{T, \bar{\phi}}(S \cup\{i\})=v_{T, \bar{\phi}}(S)$ for all $S \subseteq T$, and so $i \in N P\left(v_{T, \bar{\phi}}\right)$.

To show the other direction, now suppose that $i \in N P\left(v_{T, \bar{\phi}}\right)$ and that there exists $j \in(B \cup S) \backslash T$ such that $v(\{i, j\}) \neq 0$. Then $v_{T, \bar{\phi}}(\{i\})=v(\{i\} \cup$ $((B \cup S) \backslash T))-\sum_{k \in(B \cup S) \backslash T} \bar{\phi}_{k}\left(v^{\{i\} \cup((B \cup S) \backslash T}\right) \neq 0$, which contradicts both $i \in N P\left(v_{T, \bar{\phi}}\right)$ and $v_{T, \bar{\phi}} \in \mathcal{G}_{a}$. So, there exists no $j \in(B \cup S) \backslash T$ such that $v(\{i, j\}) \neq 0$, that is, $i \in N P(v)$.

Solution $\bar{\phi}$ being different from the Shapley value on the class of assignment games follows from the following example.

Example 3.13. Consider the assignment game of Example 3.3 which Shapley value equals $\phi^{S h}(v)=\left(\frac{1}{6}, \frac{2}{3}, \frac{7}{6}\right)$. However, $\bar{\phi}(v)=\left(\frac{2}{3}, \frac{2}{3}, \frac{2}{3}\right)$.

From Proposition 3.12 and Example 3.13 , it follows that the mentioned (in Subsection 2.1) approaches of Hart and Mas-Colell are not valid on $\mathcal{G}_{a}$.

\section{An axiomatization of the Shapley value for assignment situations}

Given an assignment situation with buyer-seller partition $(B, S)$, consider the communication graph on $N$ in which the links reflect all matching possibilities. So, the graph on $N$ is the complete bipartite graph $L^{B, S}=\{\{i, j\} \subseteq$ 
$N \mid i \in B, j \in S\}$. Since every coalition that contains at least one seller and at least one buyer is connected in $L^{B, S}$, and all coalitions that contain only buyers or only sellers have worth zero in the assignment game $v_{A}$, for every graph restricted assignment game $\left(v_{A}, L^{B, S}\right), A \in \mathcal{A}^{B, S}$, it holds that the Myerson restricted game $\left(v_{A}\right)^{L^{B, S}}$ is equal to $v_{A}$, and thus, an assignment game $v_{A}$ is a graph game on the corresponding complete bipartite graph.

A general bipartite graph on $(B, S)$ is a graph $L \subseteq L^{B, S}$ with $\{i, j\} \in L$ only if $i \in B$ and $j \in S$ or vice versa. We denote the class of all bipartite graphs on $(B, S)$ by $\mathcal{L}^{B, S}$. Typically, a matching is a bipartite graph that is not complete. Note that if for an assignment situation $A \in \mathcal{A}^{B, S}$ it holds that $v_{A}=\left(v_{A}\right)^{L}$ for some bipartite graph $L \in \mathcal{L}^{B, S}$, then also $v_{A}=\left(v_{A}\right)^{L^{\prime}}$ for every bipartite graph $L^{\prime} \supset L$. The minimal bipartite graph $L_{A}^{m}$ such that $v_{A}=\left(v_{A}\right)^{L_{A}^{m}}$ is $L_{A}^{m}=\left\{\{i, j\} \subset N \mid i \in B, j \in S\right.$ and $\left.a_{i, j}>0\right\}$.

Although not explicitly written, Theorem 2.1 (i) holds more general in the sense that component efficiency and graph game fairness characterize the Myerson value on any restricted class of graph games $\mathcal{G}^{N} \times \mathcal{C}$ such that $\mathcal{C} \subseteq \mathcal{L}^{N}$ is comprehensive, that is, for any $L \in \mathcal{C}$ and $L^{\prime} \subseteq L$ it holds that $L^{\prime} \in \mathcal{C}$. For example, the class $\mathcal{L}^{B, S}$ of all bipartite graphs between the sets of buyers $B$ and sellers $S$ satisfies this property.

Next, we show that component efficiency and graph game fairness characterize the Shapley value (on the class of assignment games) when we consider this class as graph games on bipartite graphs $\mathcal{G}^{B, S} \times \mathcal{L}^{B, S}$. Instead of working on this class of graph games, we state this axiomatization directly for assignment situations ${ }^{6}$

We refer to the solution that assigns to every assignment situation $A \in \mathcal{A}^{B, S}$ the Shapley value of the corresponding assignment game $v_{A}$ as the Shapley value for assignment situations, and denote it by $\phi^{S h}(A)=\phi^{S h}\left(v_{A}\right)$.

A submarket in assignment situation $A \in \mathcal{A}^{B, S}$ is a set of buyers and sellers such that all buyers in the set have zero valuation for the goods offered by the sellers outside the set, and all buyers outside the set have zero valuation for the goods offered by sellers inside the set.

Definition 4.1. Let $A \in \mathcal{A}^{B, S}$ be an assignment situation. Then $B^{\prime} \cup S^{\prime}$, $B^{\prime} \subseteq B, S^{\prime} \subseteq S, B^{\prime} \cup S^{\prime} \neq \emptyset$, is a submarket of $A$ if $a_{i, j}=0$ for all $(i, j) \in\left(B^{\prime} \times\left(S \backslash S^{\prime}\right)\right) \cup\left(\left(B \backslash B^{\prime}\right) \times S^{\prime}\right)$.

Applied to assignment situations, component efficiency of a solution implies that the sum of the payoffs of all players in a submarket equals the worth

\footnotetext{
${ }^{6}$ Note that there is a one to one correspondence between assignment games and assignment situations in the sense that every assignment situation obviously yields a unique assignment game, but also for every assignment game the two player coalitions containing a buyer and a seller uniquely determine the assignment situation.
} 
of that submarket. Graph game fairness of a solution applied to assignment situations implies that decreasing the valuation of one particular buyer for the good offered by a particular seller to zero, changes the payoffs of this buyer and seller by the same amount.

Definition 4.2. Solution $f$ on $\mathcal{A}^{B, S}$ satisfies

- submarket efficiency, if for all $A \in \mathcal{A}^{B, S}$ and for all submarkets $\left(B^{\prime}, S^{\prime}\right)$ of $A$, it holds that $\sum_{i \in B^{\prime} \cup S^{\prime}} f_{i}(v)=v_{A}\left(B^{\prime} \cup S^{\prime}\right)$,

- valuation fairness, if for every buyer $i \in B$, every seller $j \in S$ and every pair of assignment situations $A, \bar{A} \in \mathcal{A}^{B, S}$ such that $\bar{a}_{i, j}=0$ and $a_{g, h}=\bar{a}_{g, h}$ for all $(g, h) \in((B \backslash\{i\}) \times S) \cup(B \times(S \backslash\{j\}))$, it holds that $f_{i}(A)-f_{i}(\bar{A})=f_{j}(A)-f_{j}(\bar{A})$.

Next, similar to the proof of Theorem 2.1. (i) (see Myerson [13]) the following can be shown.

Theorem 4.3. The Shapley value $\phi^{\text {Sh }}$ is the unique solution for assignment situations that satisfies submarket efficiency and valuation fairness.

Proof. We first prove that the Shapley value satisfies the two axioms.

(i) Note that $\left(B^{\prime}, S^{\prime}\right)$ being a submarket in the assignment situation $A \in \mathcal{A}^{B, S}$ implies that $C=B^{\prime} \cup S^{\prime}$ is a game-component in $v_{A}$, that is, $v_{A}(T)=$ $v_{A}(T \cap C)+v_{A}(T \backslash C)$ for all $T \subseteq B \cup S$. The Shapley value for assignment situations satisfying submarket efficiency then follows from the Shapley value for TU-games satisfying component efficiency, that is, $\sum_{i \in C} \phi_{i}^{S h}(v)=v(C)$ for every game-component $C$ in any game $v \in \mathcal{G}^{N}$, see Chang and Kan [3].

(ii) If $a_{g, h}=\bar{a}_{g, h}$ for all $(g, h) \in((B \backslash\{i\}) \times S) \cup(B \times(S \backslash\{j\}))$, then all coalitions which worth in $v_{A}$ is different from its worth in $v_{\bar{A}}$ should contain both players $i$ and $j$. So, $i$ and $j$ are symmetric players in $v_{A}-$ $v_{\bar{A}}$. The Shapley value for assignment situations satisfying valuation fairness then follows from the Shapley value for TU-games satisfying fairness (see Subsection 2.1).

We prove uniqueness $5^{7}$ by induction on the number of non-zero valuations $k(A)=|K(A)|$, where $K(A)=\left\{(i, j) \in B \times S \mid a_{i, j}>0\right\}$. Suppose that $f: \mathcal{A}^{B, S} \rightarrow \mathbb{R}^{N}$ satisfies submarket efficiency and valuation fairness. If $k(A)=0$, then all singleton player sets form a submarket, and thus $f(A)$ is determined (uniquely) by submarket efficiency.

\footnotetext{
${ }^{7}$ This goes along similar lines as Myerson [13. proves uniqueness of the Myerson value for graph games by induction on the number of links $|L|$.
} 
Proceeding by induction, assume that $f\left(A^{\prime}\right)$ is (uniquely) determined whenever $0 \leq k\left(A^{\prime}\right)<k(A)$. Take any submarket $C=B^{\prime} \cup S^{\prime}, B^{\prime} \subseteq B, S^{\prime} \subseteq S$. If $|C|=1$ then submarket efficiency determines $f_{i}(A)$ for $i \in C$. If $|C|=2$, then $f_{i}(A), i \in C$, is determined (uniquely) by submarket efficiency and valuation fairness.

Otherwise, if $|C|>2$, then $C$ is connected in $L_{A}^{m}$. Then for all $(i, j) \in$ $(C \cap B) \times(C \cap S)$ such that $a_{i, j}>0$, let $A^{i, j} \in \mathcal{A}^{B, S}$ denote the matrix where for all $(k, l) \in B \times S$ :

$$
a_{k, l}^{i, j}=\left\{\begin{array}{ll}
0, & \text { if }(k, l)=(i, j) \\
a_{k, l} & \text { otherwise }
\end{array} .\right.
$$

From valuation fairness it follows that for all $(i, j) \in(C \cap B) \times(C \cap S)$ such that $a_{i, j}>0$,

$$
f_{i}(A)-f_{i}\left(A^{i, j}\right)=f_{j}(A)-f_{j}\left(A^{i, j}\right) .
$$

Since $C$ is a component, there is a tree $T \subseteq L_{A}^{m}(C)$ connecting all nodes in $C$. Since the values $f_{i}\left(A^{i, j}\right)$ and $f_{j}\left(A^{i, j}\right)$ are determined by the induction hypothesis, taking the equations (3) for the buyer-seller pairs $(i, j)$ such that $(i, j) \in T$, yields $|C|-1$ linear independent equations in the $|C|$ unknown payoffs $f_{i}(A), i \in C$. Together with the equation $\sum_{i \in C} f_{i}(A)=v_{A}(C)$ which follows from submarket efficiency, the $|C|$ unknown payoffs $f_{i}(A), i \in C$, are uniquely determined. Since this can be done for all submarkets, the payoffs $f(A)$ are uniquely determined.

Note that from the proof it follows that the Shapley value satisfies an even stronger valuation fairness property which states that changing the valuation of one particular buyer for the good offered by a particular seller in any way, changes the payoffs of this buyer and seller by the same amount. Again this follows from fairness (see Subsection 2.1) of the Shapley value and the fact that this buyer and seller are symmetric players in the difference game. However, for the characterization the weaker version looking at valuation zero is sufficient.

The Shapley value for assignment situations also satisfies a stability property similar to that of the Myerson value. This property states that the payoffs of buyer $i$ and seller $j$ do not decrease if we only increase the valuation of buyer $i$ for the good offered by seller $j$.

Theorem 4.4. Consider assignment situations $A, \bar{A} \in \mathcal{A}^{B, S}$ such that for some $i \in B, j \in S$ it holds that $\bar{a}_{i, j} \geq a_{i, j}$, and $a_{g, h}=\bar{a}_{g, h}$ for all $(g, h) \in$ $((B \backslash\{i\}) \times S) \cup(B \times(S \backslash\{j\}))$. Then $\phi_{i}^{S h}(\bar{A}) \geq \phi_{i}^{S h}(A)$ and $\phi_{j}^{S h}(\bar{A}) \geq \phi_{j}^{S h}(A)$. 
Proof. This theorem follows straightforward from the marginal contributions of the players in the corresponding assignment games, that is, from the Shapley value satisfying strong monotonicity (see Subsection 2.1).

Take assignment situations $A, \bar{A} \in \mathcal{A}^{B, S}$ such that for some $i \in B, j \in S$ it holds that $\bar{a}_{i, j} \geq a_{i, j}$, and $a_{g, h}=\bar{a}_{g, h}$ for all $(g, h) \in((B \backslash\{i\}) \times S) \cup(B \times$ $(S \backslash\{j\}))$.

Take any $T \subseteq N$ with $i \in T$. If $j \notin T$, then $v_{\bar{A}}(T)=v_{A}(T)$ and $v_{\bar{A}}(T \backslash\{i\})=$ $v_{A}(T \backslash\{i\})$, and thus $m_{i}^{T}\left(v_{\bar{A}}\right)=m_{i}^{T}\left(v_{A}\right)$. On the other hand, if $j \in T$, then $v_{\bar{A}}(T) \geq v_{A}(T)$ and $v_{\bar{A}}(T \backslash\{i\})=v_{A}(T \backslash\{i\})$, and thus $m_{i}^{T}\left(v_{\bar{A}}\right) \geq m_{i}^{T}\left(v_{A}\right)$. Since this holds for all $T \subseteq B \cup S, i \in T$, strong monotonicity of the Shapley value for TU-games implies that $\phi_{i}^{S h}(\bar{A}) \geq \phi_{i}^{S h}(A)$. Similar it follows that $\phi_{j}^{S h}(\bar{A}) \geq \phi_{j}^{S h}(A)$.

We conclude by remarking that the axiomatization provided in Theorem 4.3 also holds when we consider all assignment situations, that is, assignment situations with arbitrary sets of buyers and sellers.

\section{References}

[1] Böhm-Bawerk, E. von: Positive theory of capital (translated by W. Smart), G.E. Steckert, New York, (1923, original publication 1891)

[2] van den Brink, R.: An axiomatization of the Shapley value using a fairness property, International Journal of Game Theory 30, 309-319. (2001)

[3] Chang, C., and Kan, C-Y.: A Study on Decomposable Convex Games, Games and Economic Behavior 7 35-38. (1994)

[4] Chun, Y.: A New Axiomatization of the Shapley Value, Games and Economic Behavior 1, 119-130. (1989)

[5] Chun, Y.: On the Symmetric and Weighted Shapley Values, International Journal of Game Theory 20, 183-190. (1991)

[6] Dubey, P.: On the uniqueness of the Shapley value, International Journal of Game Theory 4, 131-139. (1975)

[7] Einy, E.: The Shapley value on some lattices of monotonic games, Mathematical Social Sciences 15, 1-10. (1988)

[8] Fernàndez, S.M.: Contributions to Operations Research Games Ph.D. Thesis University of Barcelona (2005) 
[9] Hart, S., A. Mas-Colell: The Potential of the Shapley Value, The Shapley Value, Alvin E. Roth (editor), Cambridge University Press (1988), 127137.

[10] Hart, S., A. Mas-Colell: Potential, value, and consistency, Econometrica 57,589 -614. (1989)

[11] Monderer, D., D. Samet: Variations on the Shapley Value in R. Aumann, S. Hart, ed., Handbook of Game Theory with Economic Applications Volume 3, North Holland, 2055-2076. (1988)

[12] Moulin, H.: Axioms of cooperative decision making, Cambridge University Press (1988)

[13] Myerson, R.B.: Graphs and cooperation in games, Mathematics of Operations Research 2, 225-229. (1977)

[14] Neyman, A.: Uniqueness of the Shapley value, Games and Economic Behavior 1, 116-118. (1989)

[15] Peleg, B., P. Sudhölter: Introduction to the Theory of Cooperative Games, Kluwer Academic Publishers, Boston / Dordrecht / London (2003)

[16] Pintér, M.: Young's axiomatization of the Shapley value - a new proof, working paper (2012)

[17] Roth, A.E.: The Shapley Value as a von Neumann-Morgenstern Utility, Econometrica 45, 657-664. (1977)

[18] Roth, A.E.: The expected value of playing a game in A.E. Roth, ed., The Shapley Value, Cambridge University Press, 51-70. (1988)

[19] Shapley, L.S., A Value for n-Person Games, Contributions to the Theory of Games Volume II (Annals of Mathematical Studies 28, editors: Kuhn, H.W.-Tucker, A.W.) 307-317. (1953)

[20] Shapley, L.S.: A Comparison of Power Indices and a Nonsymmetric Generalization, P-5872, The Rand Corporation, Santa Monica, CA. (1977)

[21] Shapley, L.S., M. Shubik: The assignment game I: the core, International Journal of Game Theory 1, 111-130. (1972) 
[22] Young, H. P.: Monotonic Solutions of Cooperative Games, International Journal of Game Theory 14, 65-72. (1985)

[23] Young, H. P.: Individual contribution and just compensation in A.E. Roth, ed., The Shapley Value, Cambridge University Press, 267-278. (1988)

[24] Weber, R.J.: Probabilistic values for games in A.E. Roth, ed., The Shapley Value, Cambridge University Press, 101-119. (1988) 
Tinbergen Institute is the graduate school and research institute in economics of Erasmus University Rotterdam, the University of Amsterdam and VU University Amsterdam.

More TI discussion papers can be downloaded at http://www.tinbergen.nl

Tinbergen Institute has two locations:

Tinbergen Institute Amsterdam

Gustav Mahlerplein 117

1082 MS Amsterdam

The Netherlands

Tel.: +31(0)205251600

Tinbergen Institute Rotterdam

Burg. Oudlaan 50

3062 PA Rotterdam

The Netherlands

Tel.: +31(0)10 4088900

Fax: $+31(0) 104089031$

Duisenberg school of finance is a collaboration of the Dutch financial sector and universities, with the ambition to support innovative research and offer top quality academic education in core areas of finance.

DSF research papers can be downloaded at: http://www.dsf.nl/

Duisenberg school of finance

Gustav Mahlerplein 117

1082 MS Amsterdam

The Netherlands

Tel.: +31(0)20 5258579 